\title{
Single snapshot DOA estimation
}

\section{P. Häcker and B. Yang}

Chair of System Theory and Signal Processing, Universität Stuttgart, Pfaffenwaldring 47, 70550 Stuttgart, Germany

\begin{abstract}
In array signal processing, direction of arrival (DOA) estimation has been studied for decades. Many algorithms have been proposed and their performance has been studied thoroughly. Yet, most of these works are focused on the asymptotic case of a large number of snapshots. In automotive radar applications like driver assistance systems, however, only a small number of snapshots of the radar sensor array or, in the worst case, a single snapshot is available for DOA estimation.

In this paper, we investigate and compare different DOA estimators with respect to their single snapshot performance. The main focus is on the estimation accuracy and the angular resolution in multi-target scenarios including difficult situations like correlated targets and large target power differences. We will show that some algorithms lose their ability to resolve targets or do not work properly at all. Other sophisticated algorithms do not show a superior performance as expected. It turns out that the deterministic maximum likelihood estimator is a good choice under these hard conditions.
\end{abstract}

\section{Introduction}

A common problem in array signal processing is the estimation of the DOA of $M$ targets using $N$ sensors. As within automotive applications the elevation angels are less important, there are $M$ angles (azimuth) to be estimated. Together with the distances, the angels determine the position of the relevant targets uniquely relative to the host vehicle. Using this information, the car can act in an intelligent way. Two examples of such driver assistance systems are Adaptive Cruise Control (ACC) and initializing an emergency brake (Jurgen, 2006).

Classical radar systems measure their environment using a grid of distance and relative velocity cells. This representation is sparse. The linear frequency modulated continuous wave (LFMCW) principle uses frequency sweeps (ramps) to

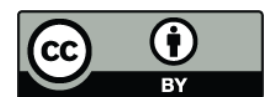

Correspondence to: P. Häcker (patrick.haecker@1ss.uni-stuttgart.de) get projections of this plane which is more efficient. Every ramp contains the targets at different frequencies, depending on its slope. Using multiple ramps, the whole plane can be reconstructed (Reiher and Yang, 2009). However, targets with similar frequencies can only be separated by their DOA.

The number of antennas and the number of snapshots are limited to get a cheap sensor. To still get acceptable results, the DOA estimator has to be as good as possible. There are many papers covering DOA algorithms using a huge amount of antennas, samples per antenna or a high SNR, respectively (Ottersten et al., 1992; Li et al., 1998; Xin and Sano, 2004; Viberg et al., 1991b; Lopes et al., 2003; Stoica and Sharman, 1990b; Gershman and Stoica, 1999), to make use of statistical asymptotic analysis. Yet, there are few papers which deal with the single snapshot case. There was a successful examination of the two classical ML algorithms (Rife and Boorstyn, 1974, 1976; Athley, 2005). However, to the knowledge of the authors, there is still no comparative study about which DOA algorithm to choose in the single snapshot case, which is what this paper is about.

There are several reasons, why a single snapshot DOA estimation is attractive in automotive radar systems:

- LFMCW ramps are designed for different ranges. In general, not all ramps are available for all distancevelocity-combinations.

- Some snapshots may be distorted by close frequency interferer and thus should not be used for angle estimation.

- Some snapshots are superpositioned by clutter. These snapshots should be avoided as well.

- The speed of reaction is enhanced by using snapshots immediately after measurement, instead of waiting for a large number of snapshots.

- The performance of DOA estimation using a single snapshot is worse than that of using multiple snapshots. The postprocessing after DOA estimation in automotive radar, namely tracking, will compensate the performance loss to a large degree. 
- The computation time is decreased as the single snapshot case allows some additional simplifications in DOA estimation.

We shortly introduce the used notation in Sect. 2 and the signal models in Sect. 3. We then take a closer look on the DOA algorithms in Sect. 4, separate them into usable and non-usable algorithms with regard to the problem at hand. In Sect. 5, we use typical automotive scenarios to simulate the performance of the DOA algorithms and conclude our work in Sect. 6.

\section{Notation}

The following notations are used in this paper: Uppercase bold letters are matrices, lowercase bold letters are vectors, letters with a hat are estimations, $(\cdot)^{\mathrm{T}}$ denotes the transposition, $(\cdot)^{\mathrm{H}}$ denotes the complex conjugate transposition, $(\cdot)^{\dagger}$ denotes the Moore-Penrose pseudo inverse, $\underset{\theta}{\operatorname{argmax}}(\cdot)$ denotes the $\boldsymbol{\theta}$ maximizing the function, $\operatorname{argmaxima}_{M}(\cdot)$ denotes the $\theta$ of the $M$ largest local maxima, $\operatorname{Tr}(\cdot)$ is the matrix trace operator, $|\cdot|$ is the matrix determinant and $\mathbf{I}$ is the identity matrix.

\section{Signal models}

Two signal models are commonly used for DOA estimation (Krim and Viberg, 1996): the deterministic model and the stochastic model. In both models, the impinging signals on the array are superpositioned by spatial and temporal white Gaussian noise $\boldsymbol{n}(t)$. In typical automotive applications, narrow band and far field conditions can be assumed to be valid. Furthermore, both signal models parametrize the sensor array and the targets' DOA by the steering matrix

$\mathbf{A}(\boldsymbol{\theta})=\left(\boldsymbol{a}\left(\theta_{1}\right), \ldots, \boldsymbol{a}\left(\theta_{M}\right)\right)$.

$\boldsymbol{a}(\theta)$ is the steering vector which can be seen as an angular transfer function. Using a uniform linear array (ULA) can reduce the signal processing effort. In general, the array can be arbitrary. For the linear array used in this paper, the steering vector can be written as

$\boldsymbol{a}(\theta)=\left(\mathrm{e}^{\mathrm{j} 2 \pi y_{1} \sin (\theta)}, \ldots, \mathrm{e}^{\mathrm{j} 2 \pi y_{N} \sin (\theta)}\right)^{\mathrm{T}}$

using the sensor positions $\boldsymbol{y}_{n}$ normalized by the wavelength. $\theta$ is defined as $0^{\circ}$ pointing to the front direction.

Let $s(t)$ be the incoming waves after mixing to baseband, the sensor array signal to be processed is given by

$\boldsymbol{x}(t)=\mathbf{A}(\boldsymbol{\theta}) \boldsymbol{s}(t)+\boldsymbol{n}(t)$.

The source signals $\boldsymbol{s}(t)$ can be deterministic or a Gaussian random process, depending on the chosen signal model. Using an LFMCW radar (Schoor and Yang, 2007) most of the targets are separated by their distance and relative velocity. The DOA estimations separate only the remaining targets. This is why most often only one or two targets need to be estimated using a single snapshot $\boldsymbol{x}(t)$.

The used array is a half wavelength minimum redundancy array (Moffet, 1968) consisting of four antennas $(M=4)$ at the positions $\boldsymbol{y}=(0,0.5,2,3)^{\mathrm{T}}$ normalized by the wavelength. As the distance between the first two antennas is half of the wavelength, the uniqueness of the DOA estimation is guaranteed. The missing redundancy leads to a high positional variance (Athley, 2005) and thus to an improved accuracy of the single target estimation compared to more conservative four antenna arrays.

\section{DOA algorithms}

The following DOA algorithms are studied in this paper: Bartlett beamformer, Multiple Signal Classification (MUSIC), Deterministic Maximum Likelihood (DML), Stochastic Maximum Likelihood (SML) and Weighted Subspace Fitting (WSF).

Since the array assumptions shift invariance, rotational invariance, special structure and the invertability of the sample correlation matrix are not fullfilled, many DOA estimation algorithms like estimators using decorrelation principles (Pillai and Kwon, 1989), Capon's beamformer (Capon, 1969), ESPRIT (Paulraj et al., 1985), Root-MUSIC (Barabell, 1983), MUD (Swindlehurst, 1991), IQML (Swindlehurst, 1991), MODE (Stoica and Sharman, 1990a) or MODEX (Gershman and Stoica, 1999) do not work and are thus not considered in this paper.

\subsection{Bartlett beamformer}

Bartlett's beamformer can be written, using the sample correlation matrix

$\hat{\mathbf{R}}=\boldsymbol{x}(t) \boldsymbol{x}(t)^{\mathrm{H}}$

as

$\hat{\boldsymbol{\theta}}_{\text {Bartlett }}=\underset{\theta}{\operatorname{argmaxima}} M\left(\frac{\boldsymbol{a}(\theta)^{\mathrm{H}} \hat{\mathbf{R}} \boldsymbol{a}(\theta)}{\boldsymbol{a}(\theta)^{\mathrm{H}} \boldsymbol{a}(\theta)}\right)$.

The Bartlett beamformer maximizes the angular spectrum in Eq. (5) and returns the position of the $M$ largest maxima as the estimates for the DOAs of the $M$ targets. An example of a beamformer spectrum is given in Fig. 1. In this scenario two $40 \mathrm{~dB}$ targets are at $0^{\circ}$ and $60^{\circ}$. Since only a single snapshot is used, the Bartlett beamformer does not show maxima at the correct DOAs. The largest maximum appears even at a DOA where no target exists.

\subsection{MUSIC}

To get the MUSIC function, an eigenvalue decomposition of $\hat{\mathbf{R}}$ must be computed. By putting the eigenvectors belonging 


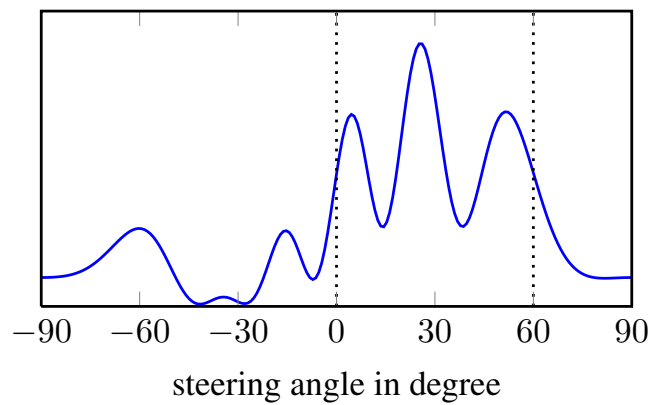

Fig. 1. Bartlett beamformer spectrum for two targets.

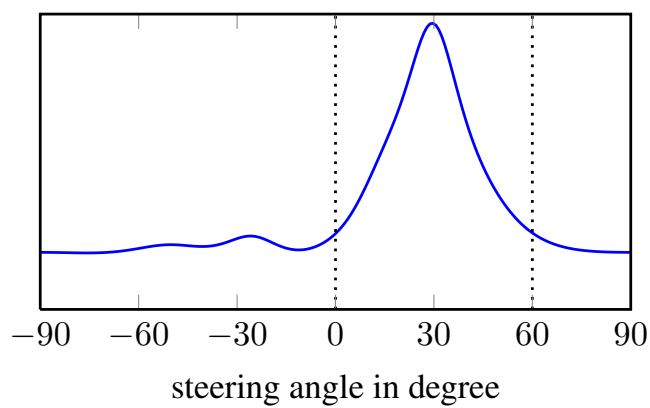

Fig. 2. MUSIC function for two targets.

to the $N-M$ weakest eigenvalues in the noise subspace matrix $\hat{\Psi}_{\text {noise }}$, the MUSIC estimator is given by (Schmidt, 1979)

$\hat{\boldsymbol{\theta}}_{\text {MUSIC }}=\underset{\theta}{\operatorname{argmaxima}} M\left(\frac{\boldsymbol{a}(\theta)^{\mathrm{H}} \boldsymbol{a}(\theta)}{\boldsymbol{a}(\theta)^{\mathrm{H}} \hat{\Psi}_{\text {noise }} \hat{\Psi}_{\text {noise }}^{\mathrm{H}} \boldsymbol{a}(\theta)}\right)$.

The MUSIC function is given in Fig. 2 using the same scenario and the same snapshot as before. MUSIC detects a non-existing target between the two real targets and it does not indicate the existence of two targets. As there is only one snapshot, the signal subspace is built up of only one eigenvector, which leads to the averaged target.

\subsection{DML}

Using the deterministic signal model, the DML approach is

$\hat{\boldsymbol{\theta}}_{\mathrm{DML}}=\underset{\boldsymbol{\theta}}{\operatorname{argmax}}\left(\operatorname{Tr}\left(\Pi_{\mathbf{A}}(\boldsymbol{\theta}) \hat{\mathbf{R}}\right)\right)$

using the projection matrix onto the column space of $\mathbf{A}(\boldsymbol{\theta})$

$\Pi_{\mathbf{A}}(\boldsymbol{\theta})=\mathbf{A}(\boldsymbol{\theta}) \mathbf{A}(\boldsymbol{\theta})^{\dagger}$

and the pseudo inverse of $\mathbf{A}(\boldsymbol{\theta})$

$\mathbf{A}(\boldsymbol{\theta})^{\dagger}=\left(\mathbf{A}(\boldsymbol{\theta})^{\mathrm{H}} \mathbf{A}(\boldsymbol{\theta})\right)^{-1} \mathbf{A}(\boldsymbol{\theta})^{\mathrm{H}}$.

Once again, a typical DML function is drawn in Fig. 3 for the same situation. $\boldsymbol{\theta}=\left(\theta_{1}, \theta_{2}\right)^{\mathrm{T}}$ is now a two-element DOA

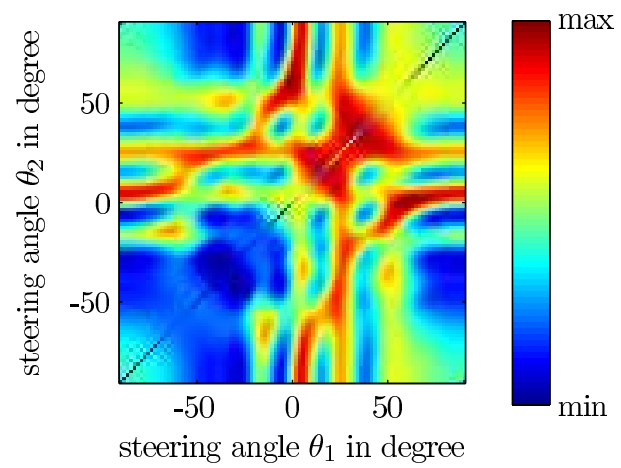

Fig. 3. DML function for two targets.

vector. While the Bartlett beamformer and MUSIC perform an one-dimensional search to estimate $\theta_{1}$ and $\theta_{2}$ of two targets, DML performs a two-dimensional search to estimate $\theta_{1}$ and $\theta_{2}$ simultaneously. Note the symmetry of the figure along its diagonal. It stems from the commutativity of the two targets, as the target numbering is arbitrary. We see from Fig. 3 that the maximum of the DML function is reached at either $\left(0^{\circ}, 60^{\circ}\right)$ or $\left(60^{\circ}, 0^{\circ}\right)$.

Besides its interpretation of the maximization of a likelihood function, Eq. (7) can be seen as maximizing the power of the input signals projected onto the model signal subspace.

\subsection{SML}

According to (Jaffer, 1988), the SML algorithm can be written as

$\hat{\boldsymbol{\theta}}_{\mathrm{SML}}=\underset{\boldsymbol{\theta}}{\operatorname{argmax}}\left(-\log \left|\mathbf{A}(\boldsymbol{\theta}) \mathbf{P}(\boldsymbol{\theta}) \mathbf{A}(\boldsymbol{\theta})^{\mathrm{H}}+\hat{\sigma}^{2}(\boldsymbol{\theta}) \mathbf{I}\right|\right)$

using the signal projection

$\mathbf{P}(\boldsymbol{\theta})=\mathbf{A}(\boldsymbol{\theta})^{\dagger}\left(\hat{\mathbf{R}}-\hat{\sigma}^{2}(\boldsymbol{\theta}) \mathbf{I}\right)\left(\mathbf{A}(\boldsymbol{\theta})^{\dagger}\right)^{\mathrm{H}}$

together with the DOA dependent noise power estimation

$\hat{\sigma}^{2}(\boldsymbol{\theta})=\frac{1}{N-M} \operatorname{Tr}\left(\Pi_{\mathbf{A}}^{\perp}(\boldsymbol{\theta}) \hat{\mathbf{R}}\right)$

and the orthogonal projection matrix

$\Pi_{\mathbf{A}}^{\perp}(\boldsymbol{\theta})=\mathbf{I}-\Pi_{\mathbf{A}}(\boldsymbol{\theta})$.

The computational effort of SML is much higher than DML, as the estimation of $\hat{\sigma}^{2}(\boldsymbol{\theta})$ is already more expensive than the whole DML approach. The scenario in Fig. 4 is again the same as before. Having a single snapshot and two targets, SML suffers numerical problems using double precision arithmetic. Some DOAs thus lead to negative logarithms. They have been interpolated by the surrounding values in Fig. 4. Nevertheless the SML function is a bit noisy which makes the optimization expensive. As a side effect of the numerical noise, the maximum of the function often leads to DOAs dominated by the noise and not to the correct ones. 


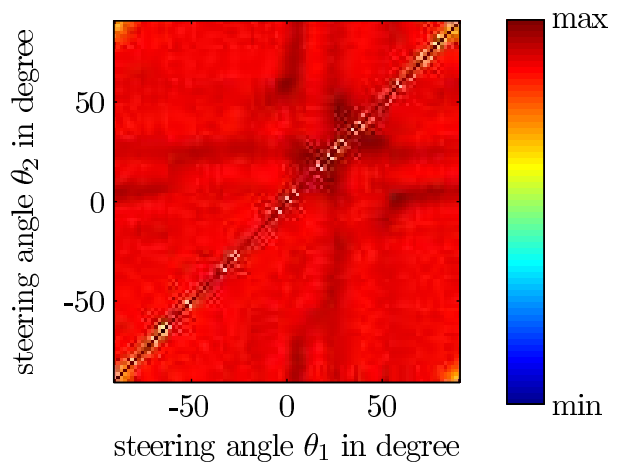

Fig. 4. SML function for two targets.

\subsection{WSF}

One approach to combine the ideas from ML and subspace based estimators is to use the signal subspace as the source of a projection into the model space, which is known as the WSF algorithm (Viberg et al., 1991a; Haykin et al., 1993). Analog to MUSIC, the eigenvalue decomposition is needed, but WSF uses the strongest eigenvalues in a diagonal matrix $\hat{\Lambda}_{\text {signal }}$ and the corresponding eigenvectors in the signal subspace matrix $\hat{\Psi}_{\text {signal }}$. WSF can then be written as

$\hat{\boldsymbol{\theta}}_{\mathrm{WSF}}=\underset{\boldsymbol{\theta}}{\operatorname{argmax}}\left(\operatorname{Tr}\left(\Pi_{\mathbf{A}}(\boldsymbol{\theta}) \hat{\Psi}_{\text {signal }} \mathbf{W} \hat{\Psi}_{\text {signal }}^{\mathrm{H}}\right)\right)$,

where $\mathbf{W}$ is a weighting matrix to reduce the impact of the subspace swap (Johnson et al., 2008a,b) defined as

$\mathbf{W}=\left(\hat{\Lambda}_{\text {signal }}-2 \hat{\sigma}^{2} \mathbf{I}+\hat{\sigma}^{2} \hat{\Lambda}_{\text {signal }}^{-1}\right)$

Instead of Eq. (12), an estimation for $\hat{\sigma}^{2}$ independent of $\theta$ can be used to decrease the computational effort.

$\hat{\sigma}^{2}=\frac{1}{N-M} \sum_{k=1}^{N-M} \hat{\Lambda}_{\text {noise }, k}$.

Note that $\hat{\mathbf{R}}$ is rank deficit in the two target case and the inversion of $\hat{\Lambda}_{\text {signal }}$ is problematic. Nevertheless it can be done with a numerically robust algorithm leading to $\mathbf{W}$ with only one element differing from zero. Again, Fig. 5 shows the WSF function using the same snapshot as before. The function has relatively broad maxima, which lead to higher variances. Besides that, it is hard to differ the true DOAs from the single or close target case at around $\left(30^{\circ}, 30^{\circ}\right)$.

\subsection{Complexity}

When comparing the computational complexity of DOA algorithms, two aspects have to be distinguished. The first two algorithms do $M$ one-dimensional optimizations, whereas

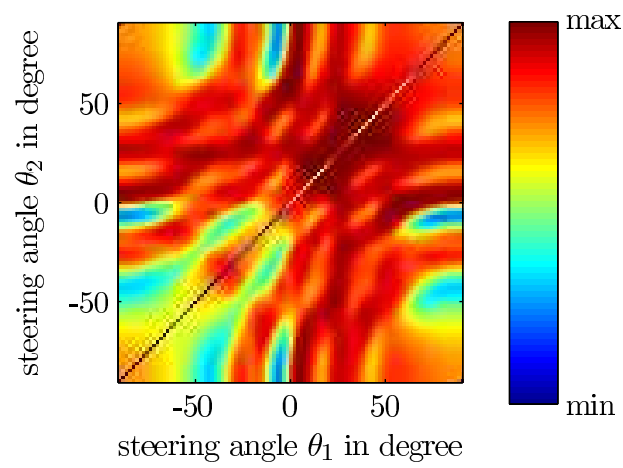

Fig. 5. WSF function for two targets.

the last three algorithms perform one $M$-dimensional optimization, which is much more expensive for large $M$. Furthermore, the effort for calculating a function value differs for different DOA algorithms. The order of the five considered DOA algorithms in this paper is roughly sorted by increasing computation time.

\section{Simulation results}

To compare the performance of different DOA algorithms, we performed Monte Carlo simulations. The signals were generated according to the signal model and the optimizations were done using a grid search followed by local optimizations.

The targets are estimated in the full angular range from $-90^{\circ}$ to $90^{\circ}$ and without any further postprocessing. The following simulation results thus show the relative performance of different DOA estimators. The absolute performance can be improved by additional processing which is not subject of this paper.

In Fig. 6 we see that for one target in front direction and 1000 Monte Carlo trials, all algorithms behave the same for medium and large SNR values regarding their root mean squared error (RMSE). The estimators are close to the deterministic Cramér-Rao Bound (CRB) which is included as an orientation. This is expected as it can be proofed, that MUSIC, DML and WSF are the same as the Bartlett beamformer for a single snapshot and a single target. For very low SNR, the threshold region is reached and thus the distance between the estimators and the CRB rapidly increases (Athley, 2005). SML has a higher probability of outliers than the other algorithms and thus a higher RMSE. With only one snapshot, SML is significantly more sensitive to noise than the other DOA algorithms in this paper.

For two targets, the situation is completely different. In Figs. 7, 8 and 9, the average RMSE of both targets is depicted. We simulated two uncorrelated widely spaced targets at $\left(0^{\circ}, 60^{\circ}\right)$ and two uncorrelated close targets at $\left(-1^{\circ}, 3^{\circ}\right)$ in Figs. 7 and 8, respectively. The mean before taking the root 


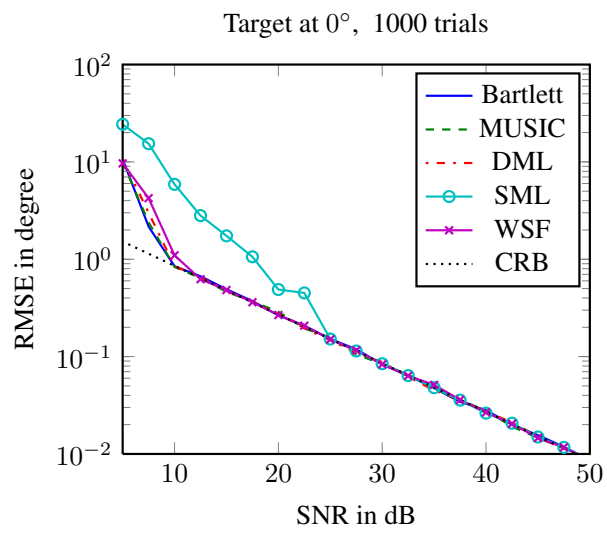

Fig. 6. DOA estimation of one target.

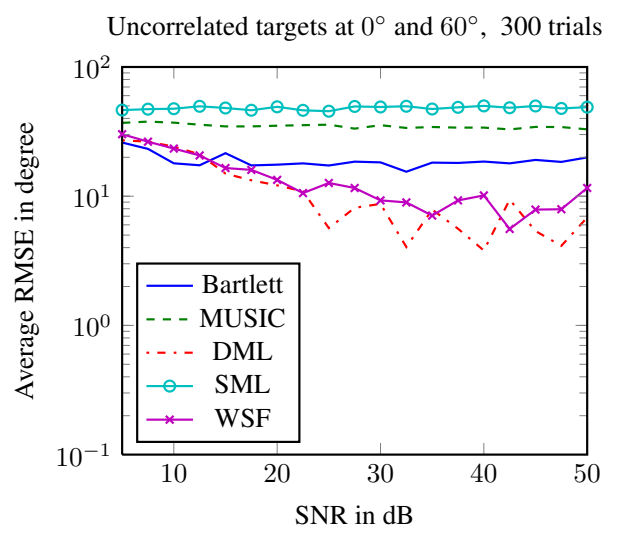

Fig. 7. DOA estimation of two widely spaced targets.

is the mean of 300 Monte Carlo trials, whereas the average after taking the root averages both targets. SML, MUSIC and the Bartlett beamformer are more or less useless. Together with their large errors, they lack an SNR dependency, which exposes a general modelling problem. WSF and DML behave better with DML being best.

In general, the average RMSE of both targets is dominated by outliers. So the estimation accuracy of most trials is much better than in the corresponding figures. There is only a fraction of trials which lead to large errors, but the fraction depends on the DOA algorithm. Figures 7 and 8 show that the probability of outliers for DML is low.

It is interesting to note that widely spaced targets are not necessarily easier to resolve than close targets, when using a sparse array. This is because the envelope of the correlation of the non-sparse array is monotonically decreasing when starting at the main lobe. As the steering vectors are less similar, the estimation is less sensitive and thus more accurate. For general arrays there is no monotonic behaviour of the envelope of the correlation function and that is why Fig. 7 does not show better results than Fig. 8.

The situation in Fig. 9 seems more challenging than in

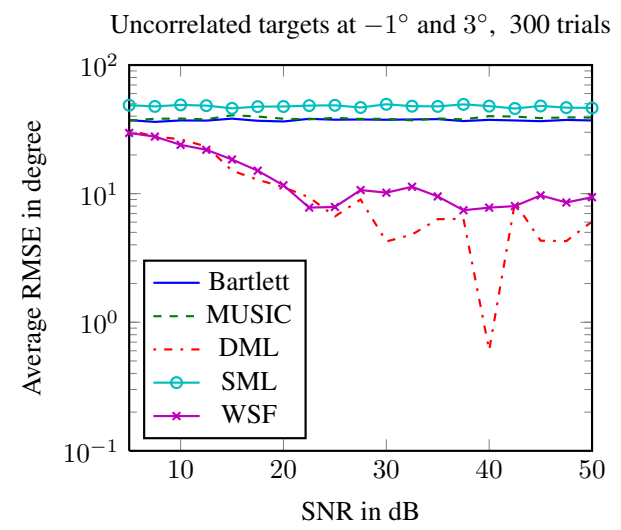

Fig. 8. DOA estimation of two close targets.

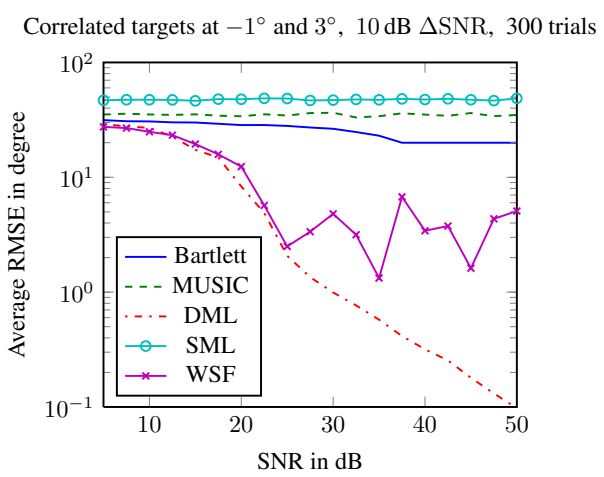

Fig. 9. DOA estimation of two differing correlated close targets.

Fig. 8. Two close targets at $\left(-1^{\circ}, 3^{\circ}\right)$ have an SNR difference of $10 \mathrm{~dB}$. One target has $5 \mathrm{~dB}$ less and the other one $5 \mathrm{~dB}$ more SNR than shown on the abscissa. The SNR difference models radar cross section fluctuations when looking at the targets from different angles. Furthermore, the phase of the signals of the two targets are fully correlated, i.e. they are always the same, which can happen when crash barrier reflections occur. This effect reduces the probability of outliers for DML and WSF and leads to an excellent DML performance. Further investigation is needed to give an exhaustive reason for that behaviour.

\section{Conclusions}

In this paper, we have compared the performance of five DOA algorithms using only a single snapshot. Many other DOA algorithms do not work at all in this case. Having only one target, the good performance of these estimators is expected, with the notable exception of SML, as the used sparse array is optimized for that case. Estimating two targets can be done with WSF and DML, but DML is both faster and more reliable. 
Acknowledgements. This research has partly been conducted with the support of the Robert Bosch Corporation, to which the authors would like to express their gratitude.

\section{References}

Athley, F.: Threshold Region Performance of Maximum Likelihood Direction of Arrival Estimators, IEEE Trans. on Signal Processing, 53, 1359-1373, 2005.

Barabell, A. J.: Improving the Resolution Performance of Eigenstructure-Based Direction Finding Algorithms, in: IEEE Proc. of Int. Conf. on Acoustics, Speech, and Signal Processing, 8, 336-339, 1983.

Capon, J.: High Resolution Frequency-Wavenumber Spectrum Analysis, IEEE Proc., 57, 1408-1418, 1969.

Gershman, A. B. and Stoica, P.: Mode with Extra-Roots (MODEX): A new DOA Estimation Algorithm with an Improved Threshold Performance, in: IEEE Proc. of Int. Conf. on Acoustics, Speech, and Signal Processing, 1999.

Haykin, S., Ho, T., Litva, J., McWhirter, J., Nehorai, A., and Nickel, U.: Radar Array Processing, Springer, 1 edn., 1993.

Jaffer, A. G.: Maximum Likelihood Direction Finding of Stochastic Sources: A Separable Solution, IEEE Proc. of Int. Conf. on Acoustics, Speech, and Signal Processing, 5, 2893-2896, 1988.

Johnson, B. A., Abramovich, Y. I., and Mestre, X.: The Role of Subspace Swap in Maximum Likelihood Estimation Performance Breakdown, IEEE Proc. of Int. Conf. on Acoustics, Speech, and Signal Processing, 2469-2472, 2008a.

Johnson, B. A., Abramovich, Y. I., and Mestre, X.: The Role of Subspace Swap in MUSIC Performance Breakdown, IEEE Proc. of Int. Conf. on Acoustics, Speech, and Signal Processing, 24732476, 2008b.

Jurgen, R. K.: Adaptive Cruise Control, SAE International, 2006.

Krim, H. and Viberg, M.: Two Decades of Array Signal Processing Research, IEEE Signal Processing Magazine, 13, 67-94, 1996.

Li, J., Stoica, P., and Liu, Z.-S.: Comparative Study of IQML and MODE Direction-of-Arrival Estimators, IEEE Trans. on Signal Processing, 46, 149-160, 1998.

Lopes, A., Bonatti, I. S., Peres, P. L., and Alves, C. A.: Improving the MODEX Algorithm for Direction Estimation, ELSEVIER's Signal Processing, 83, 2047-2051, 2003.

Moffet, A.: Minimum-Redundancy Linear Arrays, IEEE Trans. on Antennas and Propagation, 16, 172-175, 1968.
Ottersten, B., Viberg, M., and Kailath, T.: Analysis of Subspace Fitting and ML Techniques for Parameter Estimation from Sensor Array Data, IEEE Trans. on Signal Processing, 40, 590-600, 1992.

Paulraj, A., Roy, R., and Kailath, T.: Estimation of Signal Parameters via Rotational Invariance Techniques - ESPRIT, in: Proc. of Asilomar Conf. on Circuits, Systems and Computers, 83-89, 1985.

Pillai, S. and Kwon, B.: Forward/Backward Spatial Smoothing Techniques for Coherent Signal Identification, IEEE Trans. on Acoustics, Speech, and Signal Processing, 37, 8-15, 1989.

Reiher, M. and Yang, B.: Optimal Modulation Design in Linear FMCW Radar Concerning Mismatch Probability, in: Proc. of Intern. Radar Symposium (IRS), 2009.

Rife, D. C. and Boorstyn, R. R.: Single-Tone Parameter Estimation from Discrete-Time Observations, IEEE Trans. on Information Theory, 20, 591-598, 1974.

Rife, D. C. and Boorstyn, R. R.: Multiple Tone Parameter Estimation from Discrete-Time Observations, The Bell System Technical Journal, 55, 1389-1411, 1976.

Schmidt, R. O.: Multiple Emitter Location and Signal Parameter Estimation, in: Proc. RADC Spectrum Estimation Workshop, 34, 243-258, 1979.

Schoor, M. and Yang, B.: High-Resolution Angle Estimation for an Automotive FMCW Radar Sensor, in: Proc. of Intern. Radar Symposium (IRS), Cologne, Germany, 2007.

Stoica, P. and Sharman, K.: Novel Eigenanalysis Method for Direction Estimation, Radar and Signal Processing, IEE Proc. F, 137, 19-26, 1990a.

Stoica, P. and Sharman, K. C.: Maximum Likelihood Methods for Direction-of-Arrival Estimation, IEEE Trans. on Acoustics, Speech, and Signal Processing, 38, 1132-1143, 1990b.

Swindlehurst, A.: Fast Updating of Maximum Likelihood Direction of Arrival Estimates, in: Asilomar Conf. on Signals, Systems and Computers, 1, 302-306, 1991.

Viberg, M., Ottersten, B., and Kailath, T.: Detection and Estimation in Sensor Arrays Using Weighted Subspace Fitting, IEEE Trans. on Signal Processing, 39, 2436-2449, 1991a.

Viberg, M., Ottersten, B., and Nehorai, A.: Estimation Accuracy of Maximum Likelihood Direction Finding Using Large Arrays, Signals, Systems and Computers, 928-932, 1991b.

Xin, J. and Sano, A.: Computationally Efficient Subspace-Based Method for Direction-of-Arrival Estimation without Eigendecomposition, IEEE Trans. on Signal Processing, 52, 876-893, 2004. 